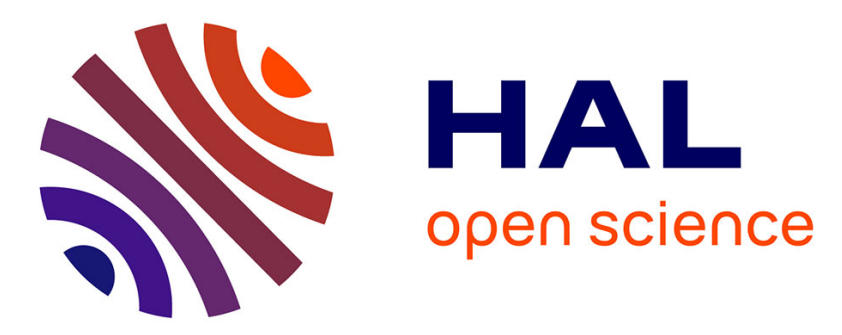

\title{
Formation of Superlattices of Gold Nanoparticles Using Ostwald Ripening in Emulsions: Transition from fcc to bcc Structure
}

Julien Schmitt, Stéphanie Hajiw, Amélie Lecchi, Jéril Degrouard, Anniina Salonen, Marianne Imperor-Clerc, Brigitte Pansu

\section{To cite this version:}

Julien Schmitt, Stéphanie Hajiw, Amélie Lecchi, Jéril Degrouard, Anniina Salonen, et al.. Formation of Superlattices of Gold Nanoparticles Using Ostwald Ripening in Emulsions: Transition from fcc to bcc Structure. Journal of Physical Chemistry B, 2016, 120 (25), pp.5759 - 5766. 10.1021/acs.jpcb.6b03287 . hal-01674448

\author{
HAL Id: hal-01674448 \\ https://hal.science/hal-01674448
}

Submitted on 2 Jan 2018

HAL is a multi-disciplinary open access archive for the deposit and dissemination of scientific research documents, whether they are published or not. The documents may come from teaching and research institutions in France or abroad, or from public or private research centers.
L'archive ouverte pluridisciplinaire HAL, est destinée au dépôt et à la diffusion de documents scientifiques de niveau recherche, publiés ou non, émanant des établissements d'enseignement et de recherche français ou étrangers, des laboratoires publics ou privés. 


\title{
Formation of Superlattices of Gold Nanoparticles
} Using Ostwald Ripening in Emulsions: Transition from $f c c$ to $b c c$ Structure

Julien Schmitt ${ }^{(1, \dagger)}$, Stéphanie Hajiw ${ }^{(1)}$, Amélie Lecchi ${ }^{(1)}$, Jéril Degrouard ${ }^{(1)}$, Anniina Salonen ${ }^{(1)}$, Marianne Impéror-Clerc ${ }^{(1)}$ and Brigitte Pansu ${ }^{(1, *)}$

${ }^{1}$ Laboratoire de Physique de Solides, UMR 8502, Bât. 510, Université Paris-Sud, F-91405 Orsay, France.

$\dagger$ current address : Physical Chemistry, Lund University, P.O. Box 124, SE-221 00 Lund (Sweden)

* corresponding author : $\quad$ brigitte.pansu@u-psud.fr Phone:XXXX

\begin{abstract}
:
An efficient method to form 3D superlattices of gold nanoparticles inside oil emulsion droplets is presented. We demonstrate that this method relies on Ostwald ripening, a well-known phenomenon occurring during the ageing of emulsions. The key point is that the nanoparticle concentration inside the smaller droplets is increasing very slowly with time thus inducing the crystallization of the nanoparticles into superlattices. Using oil-in-water emulsions doped with hydrophobic gold nanoparticles, we demonstrate that this method is efficient for different types of oils (toluene, cyclohexane, dodecane and hexadecane). 3D superlattices of the nanoparticles are obtained, with dimensions reaching a hundred nanometers. The kinetics of the crystallization depends on the solubility of the oil in water but also on the initial concentration of the gold nanoparticles in oil. This method also provides an innovative way to obtain the complete phase diagram of nanoparticles suspensions with concentration. Indeed, during this
\end{abstract}


slow crystallization process, a transition from a disordered suspension to a $f c c$ structure is observed, followed by a transition towards a $b c c$ structure. This evolution with time provides key results to understand the role played by the ligands located at the surface of the nanoparticles in order to control the type of superlattices which are formed.

Gold nanoparticles (NPs) can self-assemble in 2D or 3D periodic structures called supracrystals or superlattices. Such superlattices are expected to exhibit new physical properties due to the coupling between the NPs, compared to the case of isolated NPs in solution, ${ }^{1}$ making them of great interest in optical ${ }^{2}$, electronic ${ }^{3}$, photonic ${ }^{4}$ applications. Moreover, magnetic properties of the nanoparticles ${ }^{5}$, even gold $\mathrm{NPs}^{6}$ are also to be considered. In this context, obtaining superlattices with controlled structure and suitable sizes is an important challenge. Superlattices can form spontaneously thanks to the self-assembly of the individual nanoparticles. The organization of the superlattice formed depends not only on the shape of the nanoparticles (nanospheres ${ }^{7}$, nanocubes ${ }^{8} . .$. ) but also on the interaction between them. Nanospheres interacting only by steric repulsion maximize their configurational entropy by self-organizing in a compact structure, such as an $f c c$ lattice, as soon as the volume fraction $\varphi$ of the nanospheres reaches a critical volume fraction $\varphi^{*}$ equal to $0.494 .{ }^{7,9}$ However, preventing the particles from aggregating requires them to be coated by a ligand shell at their surface ${ }^{10}$. The softness of the shell and the entropy of the ligands change the interaction potential and as a result less compact structures can form. ${ }^{11}$

The formation of superlattices using hydrophobic gold nanoparticles stabilized by thiol groups has been already studied. ${ }^{12}$ The structure of the superlattices formed by drying a droplet of a suspension of such nanoparticles in a volatile solvent was first determined by Whetten et al. ${ }^{12 b}$ It was found that the final structure of the superlattice is greatly influenced by the size of the ligand chain compared to the radius of the gold core (ratio $\chi=\frac{L}{R_{c}}$, with $L$ the length of the stretched ligand chains and $R_{c}$ the radius of the quasi-spherical gold core). For nanoparticles with $\chi \leq 0.72$, the superlattice presents a face-centered cubic $(f c c)$ structure, while bodycentered cubic $(b c c)$ structures are found for $\chi \geq 0.75$. Several approaches implying the ligand configuration have been proposed to explain this experimental observation. ${ }^{12 \mathrm{c},}{ }^{12 \mathrm{~d}}$ Moreover, diblocs copolymers micelles in solution exhibited the same classification between hard-sphere behavior ( $f c c$ packing) and soft-sphere behavior ( $b c c$ packing) depending on the corona thickness. ${ }^{13}$ Nonetheless, it is still unknown if these observed organizations are valid for all 
volume fractions of nanoparticles $\varphi \geq \varphi^{*}$ or only when the solvent has completely evaporated. Specifically, the question of the organization at volume fractions close to $\varphi^{*}$ is still unclear.

One of the most common methods to build superlattices is through the controlled evaporation of a droplet from a colloidal suspension of nanoparticles on a substrate, allowing the formation of $2 \mathrm{D}$ or $3 \mathrm{D}$ superlattices. ${ }^{14}$ They can also form spontaneously in a dilute suspension if the attraction between the particles is enough to counterbalance the thermal energy. Abecassis et $a l .{ }^{15}$ have described the spontaneous formation of superlattices from a suspension of gold nanoparticles in toluene. Their method allows a 3D superlattice to grow within the solution. Goubet et al. ${ }^{16}$ also showed the possibility to form a supracrystalline film at the interface toluene-air. Many other methods can be used like crystallization on a liquid-liquid interface or an air-water interface. ${ }^{17}$

Recently, Lacava et al. ${ }^{18}$ used the evaporation of hexane from a hexane-in-water emulsion to form clusters of nanoparticles. However, these clusters did not exhibit a long-range order as in a superlattice. Instead of using evaporation to increase the concentration of nanoparticles inside the emulsion droplets, we have chosen to follow another approach, relying on the ageing of the emulsion itself. Several processes can destabilize an emulsion ${ }^{19}$. However through the choice of a suitable stabilizer, the droplets can be stable against coalescence and flocculation, leaving Ostwald ripening as the main destabilizing process. ${ }^{20}$ The effect of ripening is to transfer oil from the smallest droplets towards the largest ones. Indeed, because of the non-zero solubility of the oil in water, droplets exchange oil via water. This promotes the increase of the average radius of the droplets with time: smaller droplets -with a higher Laplace pressure- shrink and collapse for the benefit of the biggest ones, which are increasing in size. Eventually, the process goes on until the macroscopic phase-separation of the oil phase from the water is reached, which corresponds to the equilibrium state of the system.

In this paper, we show that the nanoparticles suspension can be concentrated and even form superlattices using Oswald ripening. The new method that we apply here, has been inspired by the approach in ${ }^{18}$ but differs in two important ways. First, starting from a more concentrated dispersion of nanoparticles in oil should lead to the formation of superlattices rather than disordered clusters. Second, replacing a direct fast evaporation process by the ageing of the 
emulsion should induce a slow increase of the concentration of the nanoparticles within the droplets, thus allowing the formation of superlattices in a controlled way.

\section{Results and Discussion}

\section{Description of the system}

The experimental system is depicted in Figure 1. It consists of oil in water emulsions containing hydrophobic gold nanoparticles of a gold core typically $2 \mathrm{~nm}$-diameter in size, stabilized by chains of dodecanethiol (see Figure 1.a). During the ripening process, inside the small droplets, the volume fraction of NPs $\varphi$ increases and, after a certain time, reaches $\varphi^{*}$, the critical volume fraction of freezing ( $50 \%$ for hard-spheres). Then a coexistence between a disordered suspension at the volume fraction $\varphi^{*}$ and domains of NPs superlattices is expected inside such droplets. With continued expulsion of oil from the droplets, superlattices should appear once the volume fraction of NPs inside them is equal to the volume fraction of melting (55\% for hard spheres). Further concentration should lead to an increase of the fraction of the crystallized domains until full crystallization. If Ostwald ripening should continue further and the NP volume fraction still increase, this would lead to a decrease of the lattice parameter or even to structural changes of the superlattice (Figure 1.b).

To estimate the volume fraction $\varphi$ occupied by the particles, we can define an apparent diameter $\left(D_{a p p}=D_{c}+2 e\right)$ as the sum of the core diameter $D_{c}$ and twice the thickness $e$ of a dense ligand corona (see Figure 1.a). This apparent diameter is estimated as $4 \mathrm{~nm}$ when $D_{c}=2 \mathrm{~nm}$, and 4.4 $\mathrm{nm}$ when when $D_{c}=2.2 \mathrm{~nm}$. The initial volume fraction of the more concentrated suspension $\left(c_{i}=20 \mathrm{wt} \%\right)$ can thus be estimated to be about $\varphi_{i}=5 \%$. With these values the final superlattice size is expected to typically range from half the size of the initial droplet as $\varphi^{*}$, the volume fraction of freezing, is expected to be around 0.5. Due to the softness of the ligand shell and as for superlattices grown using evaporation, the measured final volume fraction is expected to be larger than the classical volume fraction observed for compact hard spheres ( $74 \%$ for $f c c, 68 \%$ for $b c c$ ). For our particles, a $b c c$ packing is expected at high volume fractions of nanoparticles because of the ratio of the stretched ligand to the particle radius $\chi=\frac{L}{R_{c}}=\frac{1.6}{1.1}=1.45 \geq 0.75$.

\section{Evidence of the formation of superlattices using Ostwald ripening}


A typical cryo-TEM image of an emulsion obtained right after preparation is shown in Figure 2. This emulsion was prepared with dodecane at an initial volume fraction of $20 \mathrm{wt} \%$ of gold NPs and the initial polydispersity in the size of the oil droplets was around $25 \%$, as determined by DLS. In Figure 2, the gold NPs are homogenously dispersed inside the droplets, so we shall assume that all droplets of an emulsion present initially the same weight fraction in nanoparticles, equal to $c_{i}$.

All the emulsions containing gold NPs have been found to be stable over several months. Sedimentation of oil droplets is observed after several days or weeks, depending on the nature of the oil and the initial weight fraction of NPs. It shows that some droplets are more concentrated in NPs than initially. Indeed the density of the oil doped with NPs is larger than the density of water only for concentrations larger than $\sim 20 \mathrm{wt} \%$ for toluene, $\sim 30 \mathrm{wt} \%$ for cyclohexane and $\sim 33 \mathrm{wt} \%$ for dodecane, so for higher weight fractions than the initial ones.

The evidence of the formation of superlattices in the emulsion droplets can directly be seen using cryo-TEM. Figure 3 shows pictures recorded two months after sample preparation using toluene as suspending oil. The initial NP concentration inside the droplets was $c_{i}=5 \mathrm{wt} \%$ (Figure $3 \mathrm{a}$ ) and $c_{i}=20 \mathrm{wt} \%$ (Figure $3 \mathrm{~b}$ and $3 \mathrm{c}$ ). In Figure 3a, well-formed superlattices are observed, with several single-domains within a droplet. Their overall size is about $100 \mathrm{~nm}$, and the singledomains extend over 40 to $90 \mathrm{~nm}$. For the more concentrated sample ( $c_{i}=20 \mathrm{wt} \%$ ), we observe a coexistence between droplets where the supracrystalline order is well-defined (3b), and others where nanoparticles do not exhibit a periodic packing (3c). The existence of superlattices is evidenced by the anisotropy in the Fourier transform of the droplets (see insets in Figure 3). Nonetheless, the determination of the structure and the lattice parameters of the crystalline structures in time have been mainly carried out by SAXS.

\section{Role of the oil and the effect of its solubility in water}

During Ostwald ripening, oil is transferred from the smallest droplets to the larger ones through the water phase. The ripening process kinetics is thus mainly controlled by the oil solubility in water and can be varied enormously just buy changing the oil. The process was theoretically described by Lifshitz and Slyozov ${ }^{20}$. The typical time for the exchange of oil between the droplets and the water can be estimated by the following equation: 


$$
\frac{V}{\tau} \sim \frac{\gamma V_{m}^{2} s D}{k T}
$$

where $V$ is the droplet volume, $\tau$ the characteristic time of ripening, $\gamma$ is the interfacial tension between the particle and the water, $V_{m}$ is the molar volume of the oil, $s$ the oil solubility and $D$ the diffusivity of the oil in water, $k$ the Boltzmann constant and $T$ the temperature. The oilP123-water surface tension has been measured for dodecane $(7.9 \mathrm{mN} / \mathrm{m})$. The main difference between the given oils is their solubility in water: $5.610^{-3} \mathrm{M}$ for toluene, $6.510^{-4} \mathrm{M}$ for cyclohexane, $210^{-8} \mathrm{M}$ for dodecane and $810^{-11} \mathrm{M}$ for hexadecane. For pure oil, $\tau$ is typically a few seconds for toluene, a few minutes for cyclohexane, one month for dodecane and a few years for hexadecane. As the other parameters are quite close for all the studied oils, their solubility is thus the key parameter that controls the kinetics of the emulsion ripening.

These differences in the evolution of emulsions containing gold nanoparticles suspended in various oils are further experimentally evidenced on Figure 4, which shows SAXS spectra obtained one month after preparation for three emulsions containing the same amount of NPs $\left(D_{c}=2.2 \mathrm{~nm}, c_{i}=10 \mathrm{wt} \%\right)$ in three different oils: toluene, dodecane and hexadecane. The scattering curves have been rescaled in intensity for clarity. The three signals exhibit an increase at low angles due to the emulsion droplets and are all clearly different at larger angles. In hexadecane, the signal is dominated by the NP form factor combined with a structure factor of hard spheres with a radius $R_{H S}=2.0 \pm 0.1 \mathrm{~nm}$ and a volume fraction $\varphi_{H S}=2 \%$, (dotted line). This corresponds well to the initial concentration of the NPs, showing that, as expected, no important evolution happened with hexadecane due to its very low solubility in water. In contrast, the sample in toluene presents a series of Bragg peaks superimposed to a broad interaction peak that corresponds to a volume fraction of $\varphi_{H S}=48 \pm 1 \%$, associated with a hardsphere radius $R_{H S}=1.7 \pm 0.1 \mathrm{~nm}$. Both signals, Bragg peaks and broad peaks, are associated to nanoparticles with the same gold core size (no variation of the nanoparticles size with time). This shows that Ostwald ripening has allowed the nanoparticle's concentration within the droplets to reach the crystallization volume fraction $\varphi^{*}$ for this sample. The superlattice structure is $b c c$ with a lattice parameter equal to $4.27 \mathrm{~nm}$. In dodecane, a broad peak appears, revealing interactions between the NPs. This broad peak can be fitted using a hard-sphere interaction potential $\left(R_{H S}=2.1 \pm 0.1 \mathrm{~nm}\right.$ and $\left.\varphi_{H S}=13 \%\right)$ showing that the nanoparticle's volume fraction has increased due to Ostwald ripening. Nevertheless, the crystallization concentration in dodecane is reached only after at least one year. 
Let us note that the fitting of the SAXS data gives a hard-sphere radius of interaction $R_{H S}$ always slightly smaller than the apparent radius estimated as $2.2 \mathrm{~nm}$, whatever the oil used. This trend is in agreement with the soft nature of the ligand shell that may interpenetrate between two particles or be largely deformed. This softness is expected to influence considerably the interactions between two nanoparticles and even the nature of the superlattices that can be formed using these objects.

The solubility of oil has hence a great influence on the kinetics. In one month, the sample in toluene has almost reached its final state, whereas the sample in dodecane is still evolving, and the sample in hexadecane has remained in its initial state. It is then possible, by a careful choice of the oil used, to completely stop Ostwald ripening (using hexadecane which has a very low solubility in water) or reversely to favor it in order to reach quickly the final state of the emulsions (using toluene).

\section{Ageing of emulsions, role of the osmotic pressure}

It is known that when insoluble molecules or particles are added to the oil, Ostwald ripening is slower than for pure oil and can be ultimately stopped due to the apparition of an osmotic pressure in the droplets that opposes the ripening. ${ }^{21}$ The driving force for the ripening in a droplet with radius $R$ is proportional to $\delta P=P(R)-\Pi(c)$, where $P(R)$ is the Laplace pressure linked to the surface tension and $\Pi(c)$ is the osmotic pressure that depends on the NPs concentration $c$. Webster and Cates $^{21}$ have derived the condition of stability for an emulsion containing particles that are insoluble in the water phase, taking into account the polydispersity in the droplets' size distribution. This condition simply corresponds to a balance between the Laplace pressure (that favors Ostwald ripening) and the osmotic pressure (that prevents it) within a droplet. This allows the estimation of the final mean radius and the final volume fraction of the emulsion from the initial mean radius $R_{i}$ and initial volume fraction $\varphi_{i}$. In order to estimate what would be the final mean radius $R$ of the droplets and the final concentration, we take the expression of the Laplace pressure, $P(R)=\frac{2 \gamma}{R}$, with $\gamma$ the oil-P123-water surface tension, measured to be equal to $7.9 \mathrm{mN} / \mathrm{m}$ for dodecane. This expression is valid only when the droplets are spherical and so not when they are fully crystallized into superlattices. For the osmotic pressure, we use the Carnahan-Starling approximation, valid for hard-spheres in diluted or concentrated regime ${ }^{22} \Pi(R)=\frac{\varphi(R) k_{b} T}{\frac{4}{3} \pi R_{a p p}^{3}}\left[\frac{1+\varphi(R)+\varphi^{2}(R)-\varphi^{3}(R)}{(1-\varphi(R))^{3}}\right]$, with $\varphi(R)=\varphi_{i}\left(\frac{R_{i}}{R}\right)^{3}$ the 
volume fraction of particles in the droplets and $R_{a p p}=2 \mathrm{~nm}$ the apparent radius of the NPs. This expression is valid only when no superlattices are formed $\left(\varphi<\varphi^{*}\right)$. Since the osmotic pressure inside the small droplets increases upon ripening quicker than the Laplace pressure, the osmotic pressure could even stop the ripening before reaching the typical volume fraction of freezing $\left(\varphi^{*}\right)$. Rough estimations show that this could be the case only for the more concentrated oil in water emulsion $\left(c_{i}=20 \mathrm{wt} \%\right)$ with an initial diameter of $150 \mathrm{~nm}$, but it has not been observed.

As shown in Figure 5, a very clear effect is that when the initial concentration is larger, the ripening kinetics is much slower. This is attributed to the effect of osmotic pressure. Indeed, two weeks after preparation, the emulsion in toluene with the smallest initial NP weight fraction $(1 \mathrm{wt} \%)$ already shows the presence of a $b c c$ superlattice $\left(a_{b c c}=4.1 \mathrm{~nm}\right)$ when the one at $20 \mathrm{wt} \%$ shows only a signal corresponding to a concentrated suspension. The suspension with the intermediate concentration (10 wt\%) exhibits also a $b c c$ superlattice, but with a larger lattice parameter $\left(a_{b c c}=4.29 \mathrm{~nm}\right)$ than for $1 \mathrm{wt} \%$. All this is in agreement with the fact that the ripening process is slowed down by the presence of the NPs.

\section{Following the crystallization process upon time: from a disordered suspension to the final structure.}

The Ostwald ripening kinetics depends strongly on the nature of oil and the NP initial concentration. Crystallization thus occurs at different times depending on the oil. The shortest time is obtained for toluene, an intermediate time for cyclohexane and the longest time for dodecane. No crystallization is observed in hexadecane.

The crystallization time is also shorter if the initial NP weight fraction is lower; because the kinetics of ripening is faster. This means that it can be sufficiently slowed down to allow the experimental determination of the NP structural arrangement in the oil droplets upon time. It is therefore possible to explore in a single sample the NP phase diagram as a function of their volume fraction in the oil.

Nevertheless, at long times and for all concentrations, all the samples present the same relative Bragg peak positions, so the same supracrystalline organization which is a $b c c$ structure. This is in agreement with previous studies. ${ }^{12 \mathrm{~b}, 12 \mathrm{~d}}$ The only difference between the samples is the time 
that is necessary to wait for reaching the final state. For a gold core diameter $D_{c}=1.7 \pm 0.2 \mathrm{~nm}$, the final $b c c$ parameter is $a_{b c c}=3.8 \pm 0.1 \mathrm{~nm}$ (measured after 6 months). For $D_{c}=2.0 \pm 0.2 \mathrm{~nm}$, the final parameter is this time $4.2 \pm 0.1 \mathrm{~nm}$. From the lattice parameter, one can extract the distance $r_{N N}$ between two nearest neighbors in the superlattice: $r_{N N}=a_{b c c} \frac{\sqrt{3}}{2}$. This distance is equal to $r_{N N}=D_{c}+d_{s s}$, where $d_{s s}$ is the separation distance between two adjacent gold cores inside a region containing the ligand chains (see Figure 1.c). Interestingly, it is found that $d_{s s}$ is the same for all the NPs and is close to the value of the extended length of a unique ligand chain (1.6 $\mathrm{nm})$. The same value of $d_{s s}$ was obtained by classical evaporation.

Cryo-TEM experiments shown in Figure 3 have been performed two months after the preparation of the toluene in water emulsions with different initial NPs concentrations and one could expect that the final structure has been reached. The distances between neighboring parallel planes observed in cryo-TEM were measured for both samples. For $c_{i}=5 \mathrm{wt} \%$, this distance is equal to $3.3 \pm 0.2 \mathrm{~nm}$, whereas it is $2.9 \pm 0.2 \mathrm{~nm}$ for $c_{i}=20 \mathrm{wt} \%$. Considering that these planes belong to the (110) family of planes in the $b c c$ crystal, this gives a lattice parameter of $4.7 \pm 0.3 \mathrm{~nm}$ and $4.1 \pm 0.3 \mathrm{~nm}$ respectively. These values are in agreement to what is observed in SAXS $\left(4.2 \mathrm{~nm}\right.$ for $\left.D_{c}=2.0 \pm 0.2 \mathrm{~nm}\right)$.

The crystallization process during ripening could be followed with time using SAXS observations, before reaching the equilibrium state. In Figure 6, the results obtained for cyclohexane in water emulsions are presented. The main result is that during the ripening, nanoparticles form first a $f c c$ superlattice before rearranging into a $b c c$ one at higher NP concentrations. When the initial NP weight fraction $c_{i}$ is $20 \mathrm{wt} \%$ (gold core $2.2 \mathrm{~nm}$ ), the observed structure is $f c c$ after 8 days of ripening and becomes $b c c$ only later. When the initial $\mathrm{NP}$ weight fraction $c_{i}$ is $5 \mathrm{wt} \%$, the SAXS pattern shows the coexistence of a $f c c$ structure and a $b c c$ structure after 8 days. The different lattice parameters deduced from the diffraction patterns of Figure 6 are gathered in Table 1.

The crystallization process is quicker in toluene than in cyclohexane, so the crystallization process was followed hour by hour in a toluene in water emulsion with a $5 \mathrm{wt} \%$ initial NP weight fraction (Table 2 and Figure 7). For this batch of NPs, the gold core diameter was 1.95 $\mathrm{nm}$ with $13 \%$ polydispersity. Before crystallization, the structure factor can be described by a hard sphere model with a hard sphere diameter close to $3.9 \mathrm{~nm}$ and a volume fraction $\varphi_{H S}$ that 
will be considered as the real volume fraction. When superlattices appear, the structure factor can be modelled by the superposition of Bragg peaks and a hard sphere structure factor with a slightly smaller hard sphere diameter. The volume fraction occupied by the NPs in the crystalline part has been estimated using the apparent diameter $D_{a p p}=4 \mathrm{~nm}$ corresponding to a spherical core and a dense ligand shell.

Figure 7 shows the variation of the volume fraction upon time. Even if the values of the volume fraction strongly depend on the volume per particle that is considered, several important points can easily be raised. First, the transition from a disordered phase to a $f c c$ structure is very similar to what is expected for classical hard spheres. The fluid-to-crystal freezing transition of hard spheres was discovered more than 50 years ago in the early computer experiments. ${ }^{23}$

This transition is controlled by the translational entropy of the spheres. The accurate freezing volume fraction found for monodisperse hard spheres is 0.494 whereas the melting volume fraction is 0.545 . Indeed, we observe that for these soft spheres the values of the volume fraction for which melting and freezing are observed are close to these values and, as for hard spheres, the crystalline structure is a $f c c$ one.

When ripening goes on, the oil is expelled from the $f c c$ crystals and the volume fraction is still increasing. Due to the polydispersity of the emulsion droplets, a disordered state is still observed in coexistence. Nevertheless, contrary to a hard sphere system and due to the ligand shell softness, a transition from the $f c c$ to the $b c c$ structure is observed. ${ }^{12 \mathrm{~b},}{ }^{12 \mathrm{~d}}$ Indeed, due to the dense coverage of the gold cores with dodecanethiol chains, the Van der Waals attraction between the gold cores is mostly cancelled out, and the entropy of configuration of ligands starts to play a crucial role in the NPs' packing. It was shown recently that the ligands are less constrained in a $b c c$ packing compared to a $f c c$ one, explaining why this configuration is preferred. ${ }^{12 d, 24}$ In this study, we find that the volume fraction for which the structure changes from $f c c$ to $b c c$ is close to $68 \%$. In a $b c c$ structure, this volume fraction corresponds exactly to the concentration when the ligand shells begin to interact, when two adjacent shells just come into contact (see schemes in Figure 7). Indeed, at lower volume fractions, in the $f c c$ phase, two adjacent shells do not come into contact so the interactions are like for hard spheres. As soon as the $b c c$ structure appears, the ligand shells are able to deform more and more while the oil continues to be expelled from the crystals due to Ostwald ripening. The final state has been obtained for the 
same emulsion upon evaporation and in this final state (dotted line in Figure 7) the volume fraction is $90 \%$ and the oil is not totally expelled. The cell parameter is $a_{b c c}=4.2 \mathrm{~nm}$, the distance $r_{N N}$ between neighboring particles is thus $3.6 \mathrm{~nm}$ and the distance between gold surfaces $d_{s S}$ is therefore $1.6 \mathrm{~nm}$, a value close to $L$, the length of only one elongated ligand chain. The final bcc state appears to be controlled by the distance between two gold cores - $d_{s s}$ - that seems to be limited to the length of one elongated ligand chain. Consequently, the ligand chains are in strong interaction and adopt very constrained conformations in the final $b c c$ state.

\section{Conclusion}

In this paper we present an efficient method for the formation of 3D superlattices of gold nanoparticles, simply by allowing Ostwald ripening of an oil-in-water emulsion to concentrate the nanoparticles within the droplets. By tuning the amount of oil and of nanoparticles, one can control the size of the superlattices, which can reach hundreds of nanometers. The effect of Ostwald ripening is well demonstrated by comparing different oils and studying the samples up to one year. A key point for the formation of the superlattices is the 'slow' increase of the nanoparticle concentration within the droplets, allowing them to re-arrange and reach their equilibrium state. This method thus allows the scanning of the whole phase diagram of the system. The transition from the disordered state to the ordered one is close to what is observed for classical hard spheres and is thus linked to the translational entropy of the particles. The first structure that is observed is a $f c c$ one that transforms into a $b c c$ one when the ligand shells can interact together. This last part of the phase diagram is thus dominated by the configuration of the ligand shell. Thus the observation of a transition from a $f c c$ to a $b c c$ phase is a key result to understand the role played by the ligands in order to control the type of superlattices which are formed. In conclusion, the use of emulsions is a promising way for obtaining superlattices with controlled structures and sizes.

\section{Methods:}

\section{Sample preparation}

Hydrophobic gold nanoparticles (NPs) are synthesized using the Brust procedure ${ }^{25}$. They are composed of a gold core typically $2 \mathrm{~nm}$-diameter in size, stabilized by chains of dodecanethiol. Size and polydispersity of the NPs have been determined using Small Angle X-ray Scattering 
(SAXS) on dilute suspensions. They are grown with a good polydispersity index, which is of $13 \% .{ }^{26}$ Using thermogravimetry analysis, the amount of chains was determined and a grafting density of 6.3 ligands $/ \mathrm{nm}^{2}$ is measured, as commonly observed for such particles. ${ }^{27}$

The NPs are dispersed in the oil phase (toluene, cyclohexane, dodecane or hexadecane) at the desired initial weight concentration $\left(c_{i}=1,5,10,20 \mathrm{wt} \%\right)$ before preparing the emulsion. The oils used were purchased from Sigma-Aldrich and used as received. Droplet interfaces are stabilized by the non-ionic copolymer P123 $\left(\mathrm{EO}_{20} \mathrm{PO}_{70} \mathrm{EO}_{20}\right.$, Pluronic BASF $\left.\circledR\right)$ also used as received. The choice of the surfactant in order to successfully trap the nanoparticles within the droplets is important and has been emphasized by Lacava et $a l^{28}$. The emulsions were prepared by mixing an aqueous solution of P123, $2.5 \mathrm{wt} \%$ in water, and the oil phase containing the nanoparticles at the desired weight percent $\left(c_{i} \mathrm{wt} \%\right)$. The mixtures have been sonicated using a sonication probe (Ultrasonic processor, Bioblock Scientific, France). The weight ratio between the oil and the hydrophobic part of the surfactant (PPO part) has been fixed to 3. Experimental conditions have thus been adjusted in order to obtain droplets with an initial mean diameter of approximately $D_{0}=150 \mathrm{~nm}$ as measured by Dynamic Light Scattering (DLS). The mean number of NPs per droplet is about 100 for a $1 \mathrm{wt} \%$ suspension, 500 for $5 \mathrm{wt} \%, 1000$ for $10 \mathrm{wt} \%$ and 2200 for $20 \mathrm{wt} \%$. These numbers are thus much larger than in Lacava et al $^{18}$ and sufficient to form superlattices rather than disordered clusters.

\section{Sample characterization}

Emulsions have been analyzed using transmission electron microscopy (cryo-TEM). The emulsion was diluted three times in water before cryo-fixation and a contrast agent (ammonium molybdate, $\left.-\left(\mathrm{NH}_{4}\right)_{6}(\mathrm{Mo})_{7} \mathrm{O}_{24}, 4 \mathrm{H}_{2} \mathrm{O}-\right)$ was added in the water phase to enhance the contrast of the oil droplets with respect to water. A $3 \mu$ d drop of the sample was deposited onto a glow discharged Quantifoil R2/2 grid (Quantifoil Micro Tools, Jena, Germany) and vitrified in liquid ethane using a home-made cryo-fixation device. Frozen samples were transferred to a Gatan 626 cryo-holder (Gatan Inc., Warrendale, PA, USA) and observed at $-180^{\circ} \mathrm{C}$ with a JEM 2010 Field Emission Gun cryo-Transmission Electron Microscope or a JEOL 2010 UHR (Jeol, Tokyo, Japan) using an acceleration voltage of $200 \mathrm{kV}$ and a x50 000 magnification. Images were recorded with a Gatan Ultrascan camera with $700 \mathrm{~nm}$ to $1.5 \mu \mathrm{m}$ of defocus under low-electrondose conditions. 
Emulsions have also been studied by SAXS and DLS at different stages of the ripening process. SAXS measurements were mostly made at the ID02 synchrotron beamline (ESRF) and some additional measurements were performed either on a Nanostar Brüker instrument (IBBMC, Orsay, France) with a $q$-range $1.5 .10^{-2} \leq q \leq 0.5 \AA^{-1}$ or on a home-made instrument (MOMAC, LIONS/LPS, Orsay, France), with a molybdenum source and a $q$-range $2.10^{-2} \leq q \leq 3.2 \AA^{-1}$. Polydispersity of the emulsion droplets was estimated using Dynamic Light Scattering (DelsaNano C nanosizer from Beckman Coulter). A polydispersity of around $25 \%$ is found right after the preparation of the emulsions.

Surface tension of the interface dodecane-P123-water was measured by the pendant drop method with a Tracker apparatus from TECLIS, France.

\section{SAXS data treatment}

For SAXS modeling, the signal is the product of the form factor of the NPs and of their structure factor. The form factor of the nanoparticles has been fixed with a core radius of $1 \mathrm{~nm}$ or 1.1 $\mathrm{nm}$ (depending on the batch) and a polydispersity index of $13 \%$, in agreement with previous studies. ${ }^{26}$ The soft corona offers no contrast with the solvent and cannot be detected with SAXS. When no superlattice is recorded, interactions between the NPs inside the droplets have been described by a Percus-Yevick model for hard-spheres ${ }^{29}$, using a hard-sphere radius $\left(R_{H S}\right)$ and a particle volume fraction $\left(\varphi_{H S}\right)$. The subtle effect of the solvent on the interactions, evidenced in previous experiments, ${ }^{26}$ is not taken into account in this study, as the signal measured in emulsions does not allow measuring such refined effects and hence a hard-sphere model is enough to capture the general features of the interactions. When superlattices are formed, Bragg peaks are observed on the scattering signal. They are fitted by the diffraction of finite size crystals with either a body-centered cubic $(b c c)$ structure or a face-centered cubic $(f c c)$ one.

In all intensity curves, the emulsions droplets give a contribution at low angle, which has been modeled using the simple Porod expression for scattering from sharp interfaces ( $q$-behavior in $\left.q^{-4}\right)$

\section{Acknowledgements}


The authors thank Olivier Taché (MOMAC experiment, CEA-LIONS+LPS, funded by RTRA "Triangle de la Physique") and Dominique Durand (Nanostar experiment, IBBMC, Orsay) for their efficient and kind assistance for collecting the X-ray data. For the cryo-TEM experiments, the authors acknowledge the financial support from the French CNRS (FR3507), the CEA METSA network (www.metsa.fr) and the "Investissements d'Avenir" LabEx PALM (ANR-10LABX-0039-PALM). 


\section{Tables}

\begin{tabular}{|l|l|l|l|l|l|}
\hline$c_{i}$ & $t$ & $\begin{array}{l}a_{b c c} \\
(\mathrm{~nm})\end{array}$ & $\begin{array}{l}a_{f c c} \\
(\mathrm{~nm})\end{array}$ & $\varphi_{a p p}$ & $\begin{array}{l}d_{s s} \\
(\mathrm{~nm})\end{array}$ \\
\hline $5 \mathrm{wt} \%$ & 8 days & 4.9 & 6.2 & $\begin{array}{l}\sim 66 \%(b c c) \\
\sim 66 \%(f c c)\end{array}$ & $\begin{array}{l}2.0(b c c) \\
2.2(f c c)\end{array}$ \\
\hline $5 \mathrm{wt} \%$ & $\begin{array}{l}9 \\
\text { months }\end{array}$ & 4.35 & $/$ & $\sim 95 \%$ & 1.6 \\
\hline $20 \mathrm{wt} \%$ & 8 days & $/$ & 6.35 & $\sim 60 \%$ & 2.3 \\
\hline $20 \mathrm{wt} \%$ & $\begin{array}{l}9 \\
\text { months }\end{array}$ & 4.35 & $/$ & $\sim 95 \%$ & 1.6 \\
\hline
\end{tabular}

Table 1: Different structures and lattice parameters of the superlattices built using Ostwald ripening of NP-doped cyclohexane in water emulsions. $c_{i}$ is the initial NPs weight fraction in cyclohexane. The gold core diameter $D_{c}$ was $2.2 \mathrm{~nm}(12 \%$ polydispersity). $t$ is the maturation time. $\varphi_{a p p}$ is the volume fraction computed with the apparent diameter $D_{\text {app }}=4.2 \mathrm{~nm}$. $d_{s s}$ is the distance between two gold surfaces (see scheme in Figure 1).

\begin{tabular}{|l|l|l|l|l|l|l|l|}
\hline \multicolumn{9}{|l|}{$f_{c c}$ lattice } & \multicolumn{9}{l|}{$b c c$ lattice } \\
\hline $\begin{array}{l}t \\
(\text { day })\end{array}$ & $\begin{array}{l}a_{f c c} \\
(\mathrm{~nm})\end{array}$ & $\begin{array}{l}\varphi_{a p p} \\
(\%)\end{array}$ & $\begin{array}{l}d_{s s} \\
(\mathrm{~nm})\end{array}$ & $t$ (day) & $\begin{array}{l}a_{b c c} \\
(\mathrm{~nm})\end{array}$ & $\begin{array}{l}\varphi_{a p p} \\
(\%)\end{array}$ & $\begin{array}{l}d_{s s} \\
(\mathrm{~nm})\end{array}$ \\
\hline 1.31 & 6.3 & 54 & 2.5 & 2.73 & 4.69 & 65 & 2.1 \\
\hline 1.44 & 6.2 & 56 & 2.4 & 2.99 & 4.69 & 65 & 2.1 \\
\hline 1.67 & 6.05 & 61 & 2.3 & 3.29 & 4.65 & 66.7 & 2 \\
\hline 2.13 & 5.9 & 65 & 2.15 & 4.67 & 4.65 & 66.7 & 2 \\
\hline & & & & 6.92 & 4.64 & 67 & 2 \\
\hline & & & & 23.5 & 4.6 & 68.8 & 2 \\
\hline & & & & final & 4.26 & 87 & 1.7 \\
\hline
\end{tabular}

Table 2: Different structures and lattice parameters of the superlattices built using Ostwald ripening of NP-doped toluene in water emulsions. The initial NP weight fraction in toluene $c_{i}$ was $5 \mathrm{wt} \%$ and the gold core diameter $D_{c}$ was $1.95 \mathrm{~nm}(13 \%) . t$ is the maturation time in days. $\varphi_{a p p}$ is the volume fraction computed with the apparent diameter $D_{a p p}=4.0 \mathrm{~nm} . d_{s s}$ is the distance between two gold surfaces (see scheme in Figure 1). "final" correspond to the case of complete evaporation. 


\section{Figures}

(a) $\mathrm{Au} \mathrm{NP}$

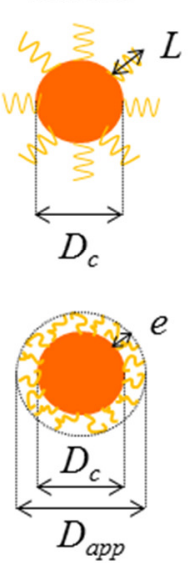

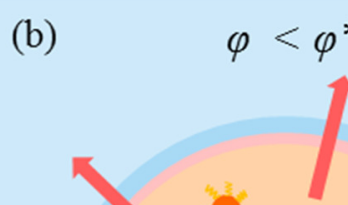
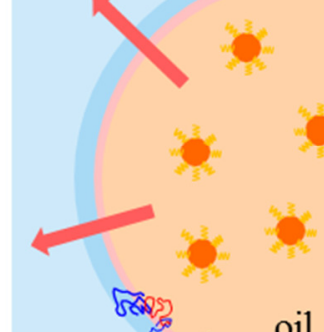

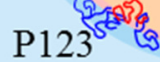
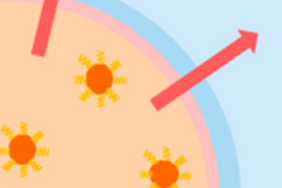

oil

$$
\varphi^{*}
$$
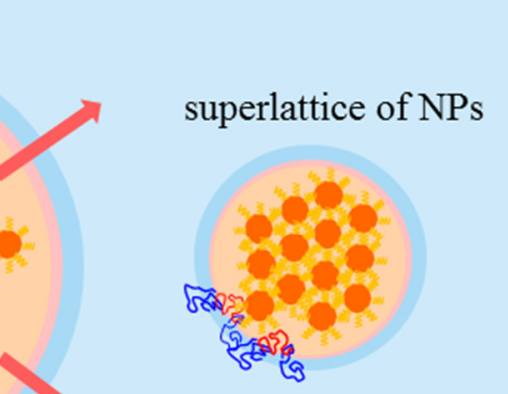

(c)

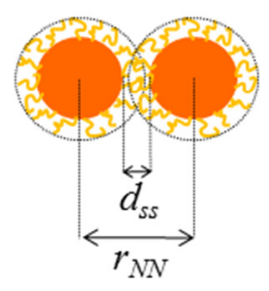

Figure 1: Scheme of the experimental system. (a) Schematic drawing of the NPs and different sizes: $D_{c}$ the diameter of the gold core, $L$ the length of a fully extended dodecanethiol chain, $D_{a p p}=D_{c}+2 e$ the apparent radius of the NP ( $e$ is the thickness of a dense corona). (b) Illustration of the Ostwald ripening and of the formation of NPs superlattices. (c) Distances between two NPs inside a superlattice: $r_{N N}$ is the distance between two gold cores, $d_{s s}$ the separation distance between two adjacent cores.

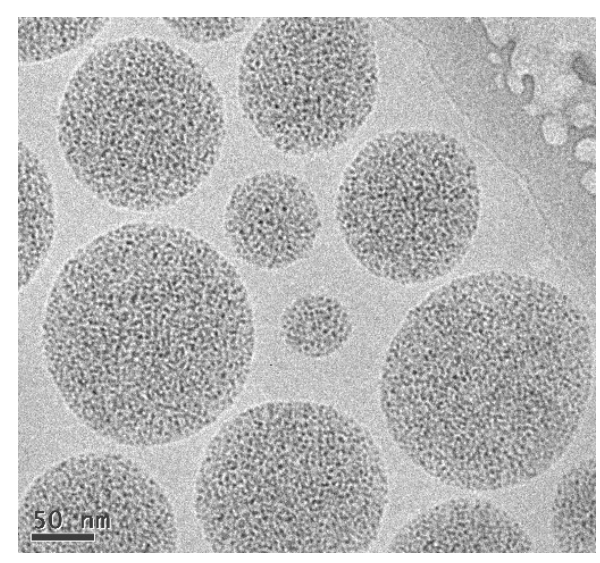

Figure 2: Cryo-TEM image of an emulsion in dodecane $\left(c_{i}=20 \mathrm{wt} \%\right)$ right after sonication. The gold nanoparticles correspond to the black dots inside the oil droplets. 

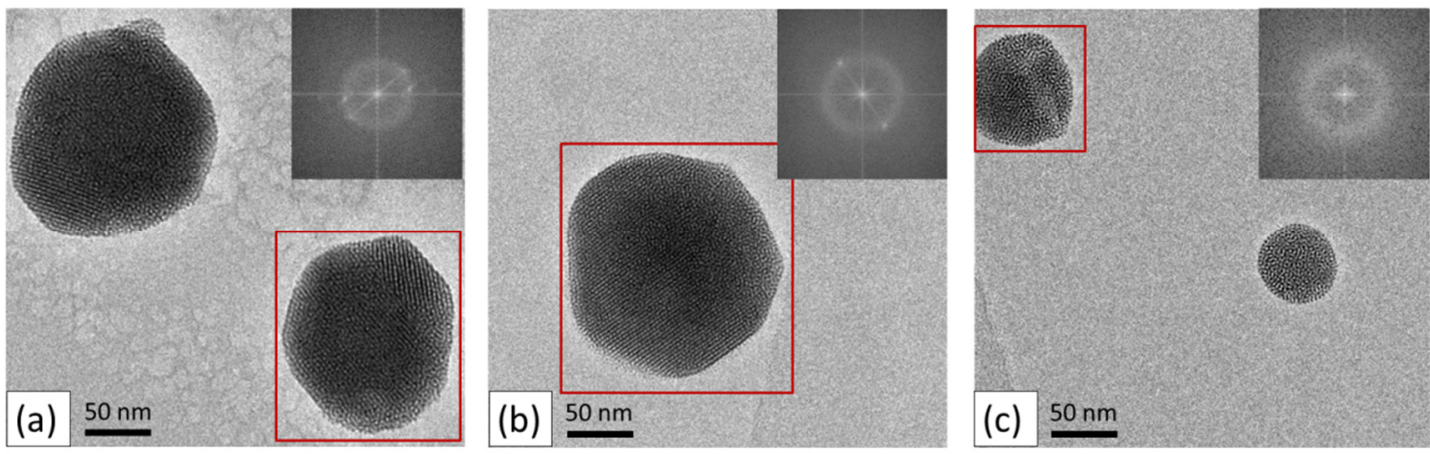

Figure 3: Cryo-TEM images of superlattices formed in emulsion droplets of toluene-in-water, measured two months after preparation. The gold core diameter is $D_{c}=2.06 \mathrm{~nm}$ and the initial NP weight fraction is $c_{i}=5 \mathrm{wt} \%$ (a) and $c_{i}=20 \mathrm{wt} \%$ (b and c). In inset, the Fourier transform of the TEM image (calculated for the region inside the red rectangle) is given.

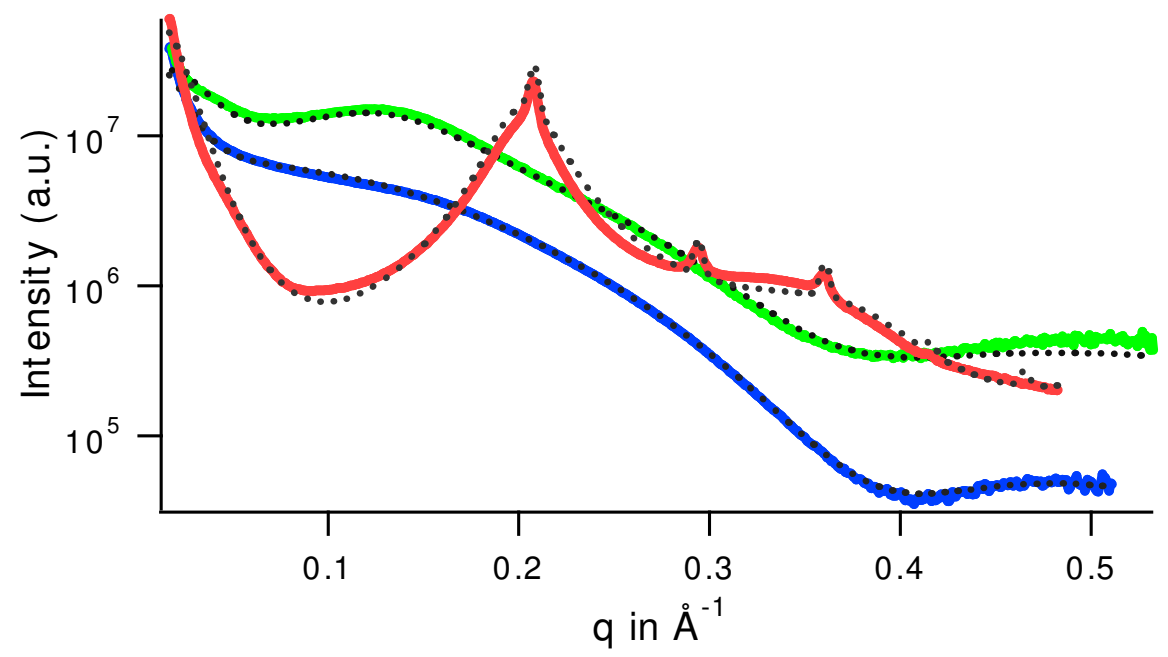

Figure 4: Comparison of SAXS spectra between emulsions in toluene (red), dodecane (green) and hexadecane (blue curve), containing the same initial NPs weight fraction $\left(c_{i}=10 \mathrm{wt} \%\right)$. Black dots are the fits of the data. All the spectra have been taken one month after the preparation of the emulsion. The form factor used is that of gold spheres with diameter $D_{c}=2.2$ $\mathrm{nm}$ and $13 \%$ of polydispersity. For hexadecane, the signal is dominated by the form factor. For dodecane, the structure factor is that of hard spheres $D_{H S}=2 \mathrm{~nm}$ at a volume fraction $\varphi_{H S}=$ $13 \%$. For toluene, the structure factor is the sum of $b c c$ Bragg peaks (lattice parameter $a=4.27$ $\mathrm{nm})$ and interacting hard spheres $\left(D_{H S}=1.7 \mathrm{~nm}, \varphi_{H S}=47 \%\right)$. 


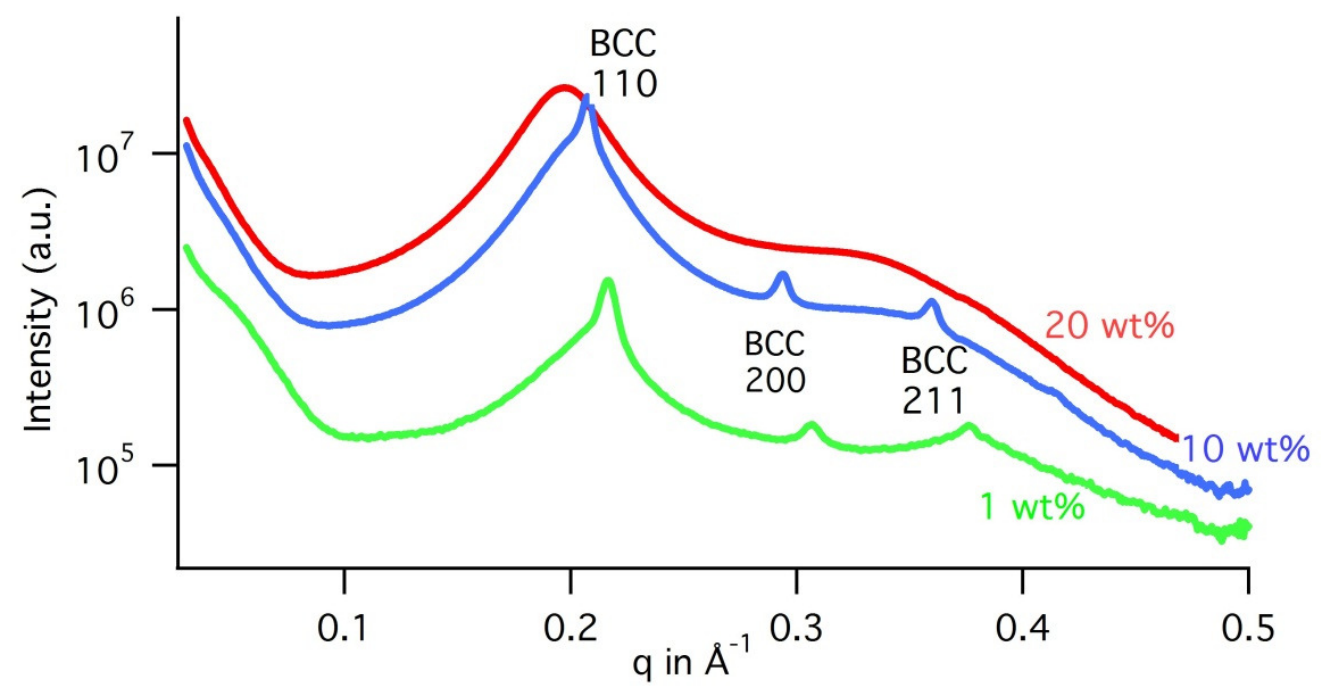

Figure 5: Effect of the initial suspension weight fraction on the Ostwald ripening. SAXS spectra of different emulsions with suspensions of NPs in toluene with different initial weight fraction are compared: $c_{i}=1 \mathrm{wt} \%$ (green curve), $c_{i}=10 \mathrm{wt} \%$ (blue) and $c_{i}=20 \mathrm{wt} \%$ (red). Measurements were performed two weeks after preparation. The lattice parameter of the bcc structure is $a_{b c c}=$ $4.1 \mathrm{~nm}$ for $1 \mathrm{wt} \%$ and $a_{b c c}=4.29 \mathrm{~nm}$ for $10 \mathrm{wt} \%$. The gold core diameter is $1.7 \mathrm{~nm}$.

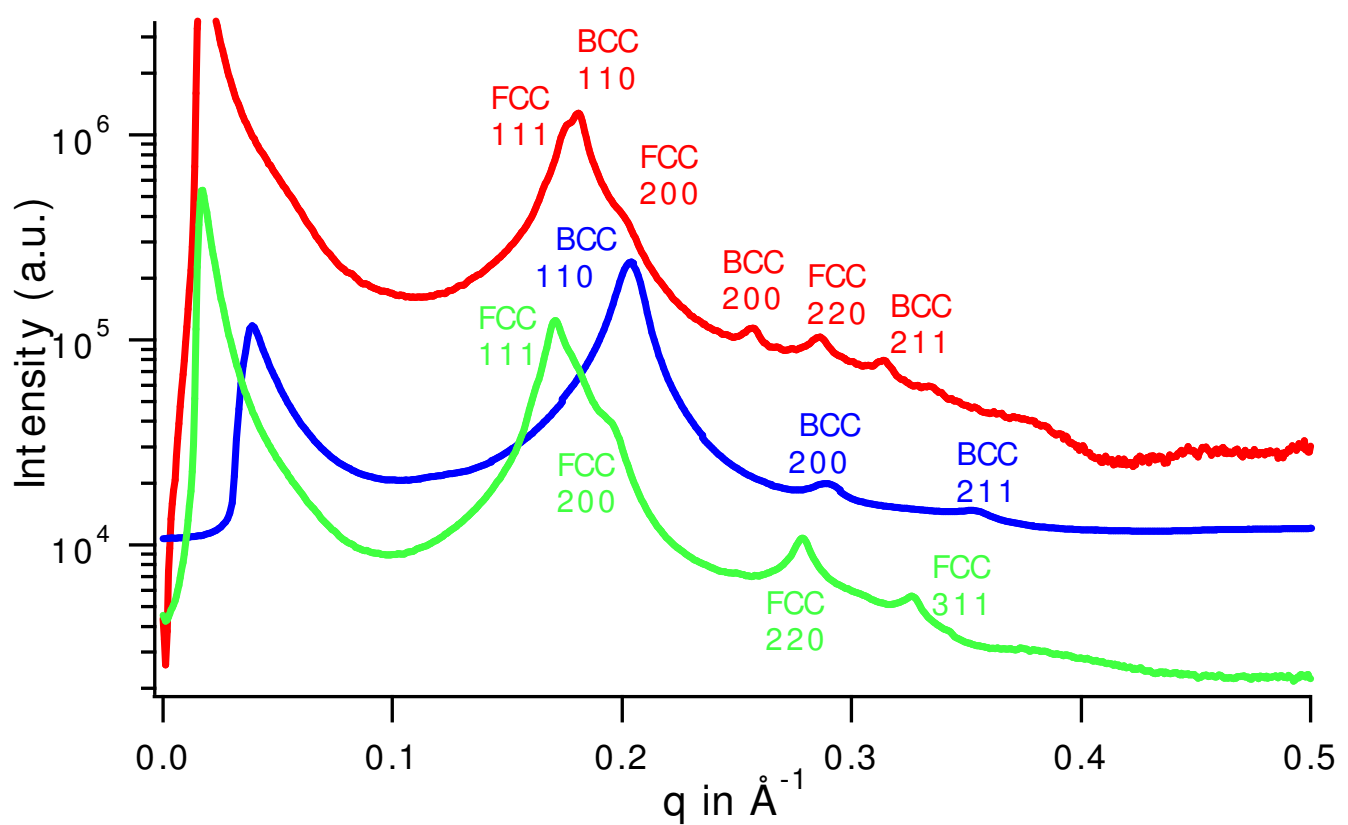

Figure 6: SAXS spectra of an emulsion in cyclohexane with $c_{i}=5 \mathrm{wt} \%$ or $20 \mathrm{wt} \%$ measured either after 8 days (red curve for $5 \mathrm{wt} \%$ and green curve for $20 \mathrm{wt} \%$ ) or 9 months of maturation (blue curve for $20 \mathrm{wt} \%$ ). The gold core diameter is $D_{c}=2.19 \mathrm{~nm}$ with $12 \%$ polydispersity. The intensities have been rescaled in order to have the three signals well separated. 


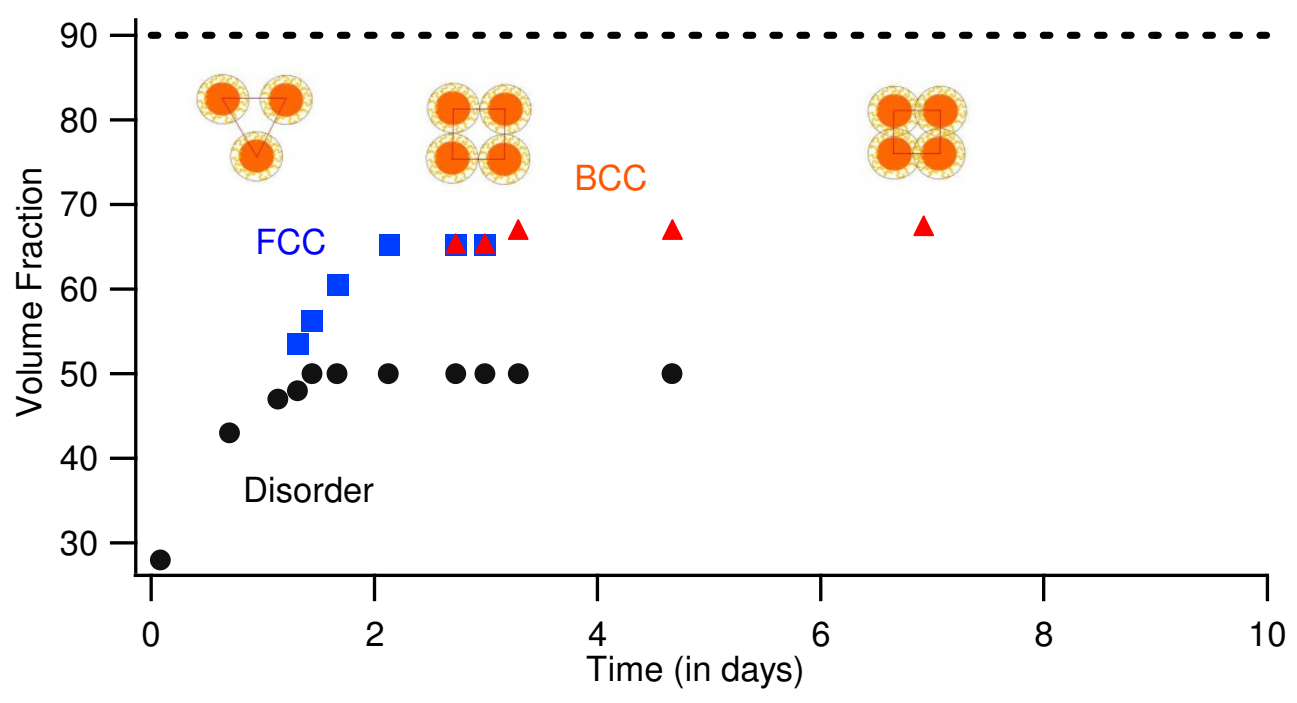

Figure 7: Phase diagram obtained by following upon time the ripening of a toluene in water emulsion with a $5 \mathrm{wt} \%$ NPs initial weight fraction. The NP volume fraction is in percentage. The gold core diameter is $1.95 \mathrm{~nm}$ with $13 \%$ polydispersity. Black circles correspond to disordered states modeled by a hard sphere potential. Blue squares correspond to $f c c$ superlattices and red triangles to $b c c$ ones. After 63 days, the volume fraction in the $b c c$ superlattice is found at $78 \%$ (point not added on the graph). The dotted line is the final volume fraction obtained by evaporating the same emulsion.

TOC Graphic

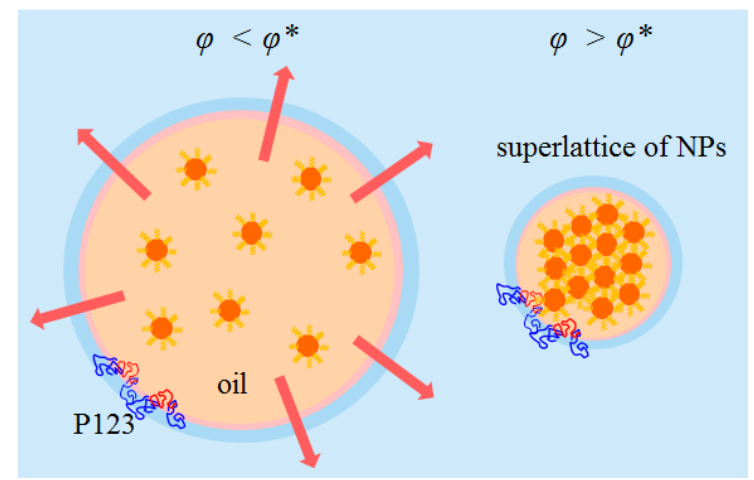




\section{References}

1. Pileni, M. P., Supracrystals of Inorganic Nanocrystals: An Open Challenge for New Physical Properties. Accounts of Chemical Research 2008, 41 (12 ), 1799-1809.

2. $\quad$ Eustis, S.; El-Sayed, M. A., Why gold nanoparticles are more precious than pretty gold: Noble metal surface plasmon resonance and its enhancement of the radiative and nonradiative properties of nanocrystals of different shapes. Chem. Soc. Rev. 2006, 35, 209-217.

3. Boettcher, S.; Strandwitz, N. C.; Schierhorn, M.; Lock, N.; Lonergan, M. C.; Stucky, G. D., Tunable electronic interfaces between bulk semiconductors and ligand-stabilized nanoparticle assemblies. Nature Materials 2007, 6, 592-596.

4. Louis, C.; Pluchery, O., Gold Nanoparticles for Physics, Chemistry and Biology. Imperial College Press: 2012.

5. Davila-Ibanez, A. B.; Salgueirino, V.; Martinez-Zorzano, V.; Marino-Fernandez, R.; Garcia-Lorenzo, A.; Maceira-Campos, M.; Munoz-Ubeda, M.; Junquera, E.; Aicart, E.; Rivas, J.; Rodriguez-Berrocal, F. J.; Legido, J. L., Magnetic Silica Nanoparticle Cellular Uptake and Cytotoxicity Regulated by Electrostatic PolyelectrolytesDNA Loading at Their Surface. ACS Nano 2012, 6 (1), 747-759.

6. Nealon, G. L.; Donnio, B.; Greget, R.; Kappler, J.-P.; Terazzi, E.; Gallani, J.-L., Magnetism in gold nanoparticles. Nanoscale 2012, 4, 5244-5258.

7. Hoover, W. G.; Ree, F. H., Melting Transition and Communal Entropy for Hard Spheres:. The Journal of Chemical Physics 1968, 49 (8), 3609-3617.

8. Demortière, A.; Launois, P.; Goubet, N.; Albouy, P.-A.; Petit, C., Shape-controlled platinum nanocubes and their assembly into two-dimensional and three-dimensional superlattices. J. Phys. Chem. B. 2008, 112, 14583-14592.

9. (a) Alder, B. J.; Wainwright, T. E., Phase Transition for a Hard Sphere System J. Chem. Phys. 1957, 27, 1208-1209; (b) Pusey, P. N.; van Megen, W.; Bartlett, P.; Ackerson, B. J.; Rarity, J. G.; Underwood, S. M., Structure of Crystals of Hard Colloidal Spheres. Physical Review Letters 1989, 63 (25), 2753-2756.

10. (a) Whetten, R. L.; Khoury, J. T.; Alvarez, M. M.; Murthy, S.; Vezmar, I.; Wang, Z. L.; Stephens, P. W.; Cleveland, C. L.; Luedtke, W. D.; Landman, U., Nanocrystal Gold Molecules. Adv. Mater. 1996, 8, 428-433; (b) Sun, S.; Murray, C. B.; Weller, D.; Folks, L.; Moser, A., Monodisperse FePt Nanoparticles and Ferromagnetic FePt Nanocrystal Superlattices. Science 2000, 287, 1989-1992; (c) Zeng, X.; Liu, F.; Fowler, A. G.; Ungar, G.; Cseh, L.; Mehl, G. H.; Macdonald, J. E., 3D Ordered Gold Strings by Coating Nanoparticles with Mesogens. Adv. Mater. 2009, 21, 1746-1750.

11. (a) Ziherl, P.; Kamien, R. D., Maximizing entropy by minimizing area towards a new principle of self-organization. J. Phys. Chem. B 2001, 105 (42), 10147-10158; (b) Choi, J. J.; Bealing, C. R.; Bian, K.; Hughes, K. J.; Zhang, W.; Smilgies, D.-M.; Hennig, R. G.; Engstrom, J. R.; Hanrath, T., Controlling Nanocrystal Superlattice Symmetry and Shape-Anisotropic Interactions through Variable Ligand Surface Coverage. J. Am. Chem. Soc. 2011, 133, 31313138; (c) Kanie, K.; Matsubara, M.; Zeng, X.; Liu, F.; Ungar, G.; Nakamura, H.; Muramatsu, A., Simple Cubic Packing of Gold Nanoparticles through Rational Design of Their Dendrimeric Corona. J. Am. Chem.Soc. 2012, 134, 808-811; (d) Gupta, S.; Camargo, M.; Stellbrink, J.; Allgaier, J.; Radulescu, A.; Lindner, P.; Zaccarelli, E.; Likos, C. N.; Richter, D., Dynamic phase diagram of soft nanocolloids. Nanoscale 2015, 7, 13924-13934.

12. (a) Goodfellow, B. W.; Rasch, M. R.; Hessel, C. M.; Patel, R. N.; Smilgies, D.-M.; Korgel, B. A., Ordered Structure Rearrangements in Heated Gold Nanocrystal Superlattices. Nano Lett. 2013, 13, 5710-5714; (b) Whetten, R. L.; Shafigullin, M. N.; Khoury, J. T.; Schaaff, T. G.; Vezmar, I.; Alvarez, M. M.; Wilkinson, A., Crystal Structures of Molecular Gold Nanocrystal Arrays. Acc. Chem. Res. 1999, 32, 397-406; (c) Landman, U.; Luedtke, W. D., 
Small is different: energetic, structural, thermal, and mechanical properties of passivated nanocluster assemblies. Faraday Discuss. 2004, 125, 1-22; (d) Goodfellow, B. W.; Yu, Y.; Bosoy, C. A.; Smilgies, D.-M.; Korgel, B. A., The Role of Ligand Packing Frustration in BodyCentered Cubic (bcc) Superlattices of Colloidal Nanocrystals. J. Phys. Chem. Lett. 2015, 6, 2406-2412.

13. Hamley, I. W.; Daniel, C.; Mingvanish, W.; Mai, S.-M.; Booth, C.; Messe, L.; Ryan, A. J., From Hard Spheres to Soft Spheres: The Effect of Copolymer Composition on the Structure of Micellar Cubic Phases Formed by Diblock Copolymers in Aqueous Solution. Langmuir 2000, $16,2508-2514$.

14. (a) Liu, S.; Zhu, T.; Hu, R.; Liu, Z., Evaporation-induced self-assembly of gold nanoparticles into a highly organized two-dimensional array. Phys. Chem. Chem. Phys. 2002, 4, 6059; (b) Goubet, N.; Richardi, J.; Albouy, P.-A.; Pileni, M.-P., Which Forces Control Supracrystal Nucleation in Organic Media? Advanced Functional Materials 2011, 21, 26932704.

15. Abécassis, B.; Testard, F.; Spalla, O., Gold Nanoparticle Superlattice Crystallization Probed In Situ. Phys. Rev. Lett. 2008, 100, 115504.

16. Goubet, N.; Portalès, H.; Cong, Y.; Afraoui, I.; Albouy, P.-A.; Mermet, A.; Pileni, M.-P., Simultaneous growths of gold colloidal crystals. J. Am. Chem. Soc. 2012, 134, 3714-3719.

17. Prasad, B. L. V.; Sorensen, C. M.; Klabunde, K. J., Gold nanoparticle superlattices. Chem. Soc. Rev. 2008, 37, 1871.

18. Lacava, J.; Born, P.; Kraus, T., Nanoparticle Clusters with Lennard-Jones Geometries. Nano Letters 2012, 12 (6), 3279-3282.

19. Binks, B. P., Modern Aspects of Emulsion Science. Royal Society of chemistry: 1998.

20. Lifshitz, I. M.; Slyozov, V. V., The kinetics of precipitation form supersaturated solid solutions. J. Phys. Chem. Solids 1959, 19, 35-50.

21. Webster, A. J.; Cates, M. E., Stabilization of emulsions by trapped species. Langmuir 1998, 14, 2068-2079.

22. Carnahan, N. F.; Starling, K. E., Equation of State for Nonattracting Rigid Spheres. $J$. Chem. Phys. 1969, 51, 635.

23. Hoover, W. G.; Ree, F. H., Melting Transition and Communal Entropy for Hard Spheres. The Journal of Chemical Physics 1968, 49, 3609.

24. Wang, Z.; Schliehe, C.; Bian, K.; Dale, D.; Bassett, W. A.; Hanrath, T.; Klinke, C.; Weller, H., Correlating Superlattice Polymorphs to Internanoparticle Distance, Packing Density, and Surface Lattice in Assemblies of PbS Nanoparticles. Nano Letters 2013, 13 (3), 1303-1311. 25. Brust, M.; Walker, M.; Bethell, D.; Schiffrin, D. J.; Whyman, R., Synthesis of thiolderivated gold nanoparticles in a two-phase liquid-liquid system. Chem. Commun 1994, 801802.

26. Hajiw, S.; Schmitt, J.; Impéror, M.; Pansu, B., Role of the Solvent in the Interaction between Gold Nanoparticles in Suspensions. Soft Matter 2015, 11, 3920-3926.

27. Hinterwirth, H.; Kappel, S.; Waitz, T.; Prohaska, T.; Lindner, W.; Lämmerhofer, M., Quantifying Thiol Ligand Density of Self-Assembled Monolayers on Gold Nanoparticles by Inductively Coupled Plasma Mass Spectrometry. ACS Nano 2013, 7 (2), 1129-1136.

28. Lacava, J.; Ouali, A.-A.; Raillard, B.; Kraus, T., On the behaviour of nanoparticles in oil-in-water emulsions with different surfactants. Soft Matter 2014, 10, 1696.

29. Percus, J. K.; Yevick, G. J., Analysis of Classical Statistical Mechanics by Means of Collective Coordinates. J. Phys. Rev. 1958, 110 (1), 1-13. 